Western University

Scholarship@Western

6-2011

\title{
VAChT knock-down mice show normal prepulse inhibition but disrupted long-term habituation
}

\author{
S. Schmid \\ Western University, sschmid8@uwo.ca \\ E. Azzopardi \\ Western University \\ X. De Jaeger \\ Western University; Universidade Federal de Minas Gerais \\ Marco Prado \\ mprado@robarts.ca \\ V. F. Prado \\ Western University, vprado@robarts.ca
}

Follow this and additional works at: https://ir.lib.uwo.ca/anatomypub

Part of the Anatomy Commons, and the Cell and Developmental Biology Commons

\section{Citation of this paper:}

Schmid, S.; Azzopardi, E.; De Jaeger, X.; Prado, Marco; and Prado, V. F., "VAChT knock-down mice show normal prepulse inhibition but disrupted long-term habituation" (2011). Anatomy and Cell Biology Publications. 92.

https://ir.lib.uwo.ca/anatomypub/92 


\section{VAChT knock-down mice show normal prepulse inhibition but disrupted long-term habituation}

Running title: Altered Habituation in VAChT -KD mice

Susanne Schmid ${ }^{1 \#}$, Erin Azzopardi ${ }^{1}$, Xavier DeJaeger ${ }^{2,3}$, Marco Prado ${ }^{1,2}$, Vania Prado ${ }^{1,2}$

${ }^{1}$ Anatomy \& Cell Biology, and ${ }^{2}$ Physiology and Pharmacology and Robarts Research Institute, Schulich School of Medicine and Dentistry, University of Western Ontario, London, ON, Canada, ${ }^{3}$ Program in Molecular Medicine, Faculdade de Medicina, UFMG, MG, Brazil

Keywords: vesicular acetyl choline transporter, startle, habituation, sensory filtering, mice, cholinergic transmission, cognitive function

August $9^{\text {th }}, 2010$

Abstract: 208

Introduction: 646

Discussion: 1259

Manuscript total: 6652

${ }^{\#}$ Corresponding author: Dr. Susanne Schmid, Dept. of Anatomy and Cell Biology, Schulich School of Medicine \& Dentistry, Medical Sciences Building, Room 470, University of Western Ontario, London, ON, N6A 5C1, Canada; phone +1-519-661 2111 ext.82668, fax: +1-519-661 3936; susanne.Schmid@schulich.uwo.ca 


\begin{abstract}
The neurotransmitter acetyl choline (ACh) plays a crucial role in both the central and peripheral nervous system. Central cholinergic transmission is important for cognitive functions and cholinergic disruptions have been associated with different neural disorders. We here tested the role of cholinergic transmission in basic cognitive functions, i.e. in prepulse inhibition (PPI) and short-term as well as long-term habituation of startle using mice with a $65 \%$ knockdown of the vesicular acetyl choline transporter (VAChT). These mice are slow in refilling cholinergic synaptic transmitter vesicles, leading to a reduced cholinergic tone. PPI has been assumed to be mediated by cholinergic projections from the midbrain to the reticular formation. Surprisingly, PPI and short-term habituation were normal in these mice, whereas long-term habituation was disrupted. This disruption could be rescued by pre-testing injections of the acetyl choline esterase inhibitor galantamine, but not by post-testing injections. The lack of a PPI deficit might be due to the fact that VAChT knock-down mice show disruptions mainly in prolonged cholinergic activity, the transient activation by prepulse processing might therefore not be sufficient to deplete synaptic vesicles. The disruption of long-term habituation indicates that the latter depends on a tonic cholinergic inhibition. Future experiments will address which cholinergic cell group is responsible for this effect.
\end{abstract}




\section{Introduction}

The neurotransmitter acetyl choline (ACh) plays a crucial role in both the central and peripheral nervous system. Disruptions in the central cholinergic system have been associated with different human disorders, including Alzheimer's disease (Felder et al., 2001, Mesulam, 2004), autism spectrum disorders (Deutsch et al.), and schizophrenia (Barak, 2009, Felder et al., 2001). One hallmark in schizophrenia is impaired prepulse inhibition (PPI) of startle. PPI is a measure of sensorimotor gating, referring to the inhibition of the startle response to a sudden intense auditory stimulus (pulse) due to prior presentation of a sub-threshold stimulus (prepulse). PPI is considered to reflect the ability to actively suppress sensory information from processing. It has been used as an assay of sensorimotor gating deficits exhibited by patients with schizophrenia, but PPI disruptions have also been reported in patients suffering from Alzheimer’s disease, Tourette’s syndrome, Huntington’s disease, or obsessive-compulsive disorder. PPI can pharmacologically be disrupted by systemic injections of muscarinic antagonists (reviewed by Barak, 2009, Jones \& Shannon, 2000b), while systemic nicotine has shown to enhance PPI in schizophrenic patients and healthy humans (Kumari et al., 2008, Kumari et al., 2001, Kumari et al., 2002, Postma et al., 2006), as well as in different animal models (Acri et al., 1994, Curzon et al., 1994, Semenova et al., 2003, Suemaru et al., 2004). PPI of startle has been proposed to be at least partly mediated by inhibitory cholinergic projections from the pedunculopontine tegmentum (PPT) and laterodorsal tegmentum (LDT) to the startle mediating neurons in the pons (Bosch \& Schmid, 2006, Bosch \& Schmid, 2008, Koch et al., 1993). Besides its descending inhibition to the caudal pontine reticular nucleus (PnC), the PPT primarily projects to higher brain structures, e.g. the thalamus, substantia nigra and ventral tegmentum dopaminergic neurons (Blaha et al., 1996, Fendt et al., 2001a, Steriade et al., 1990, Yeomans, 1995). It was suggested 
that these cholinergic PPT projections may be responsible for cortical activation and eliciting approach behaviours, while inhibiting avoidance behaviours like startle through the descending projections (Fendt et al., 2001a).

Habituation is another form of sensory filtering that is also disrupted in schizophrenia and delayed in patients suffering from autism spectrum disorders. Short-term habituation (STH) describes the decline of a behavioural response (e.g. startle) to repeated presentation of the same stimulus within a testing session, whereas long-term habituation (LTH) describes the decline of the first (or the average) response over consecutive testing sessions. Habituation is also considered being a basic form of non-associative learning. Whereas STH of startle occurs within the primary startle pathway (Davis et al., 1982, Schmid et al., 2010), LTH can be disrupted by lesions in the cerebellar vermis (Leaton \& Supple, 1986, Leaton \& Supple, 1991, Lopiano et al., 1990). Yet, there is little indication for a role of acetylcholine in habituation of startle (see Hughes, 1984).

Habituation and PPI deficits have been associated with cognitive symptoms in neural disorders. The cholinergic system plays a major role in cognitive function, and drugs facilitating cholinergic transmission have been developed as cognitive enhancers. Efficient synaptic release of ACh depends on its transport into synaptic vesicles by vesicular ACh transporter (VAChT, De Castro et al., 2009, Prado et al., 2002). Homozygous VAChT knock-down mice (VAChT $\mathrm{KD}^{\mathrm{HOM}}$ ) with a $70 \%$ reduced immunoreactivity for VAChT in the brain show decreased ability to refill synaptic vesicles (Lima Rde et al., Prado et al., 2006). VAChT KD ${ }^{\mathrm{HOM}}$ mice have a reduced capability to sustain ACh release and the injection of the cholinesterase inhibitor galantamine has previously shown to reverse motor, cardiovascular and memory deficits in mutant mice (De Castro et al., 2009, Lara et al., Prado et al., 2006). VAChT KD ${ }^{\mathrm{HOM}}$ mice are 
therefore an excellent model to study the consequences of disruptions in central cholinergic function. We here studied the effect of VAChT knock-down on basic cognitive processes such as short-term and long-term habituation as well as prepulse inhibition of startle.

\section{Material \& Methods:}

Animals: Generation and genotyping of the mice has been described before (De Castro et al., 2009, Prado et al., 2006). These mice were generated by targeting the 5' untranslated region of the VAChT gene by homologous recombination in a mixed 129S6/SvEvTac (129S6) $\mathrm{x}$ C57BL/6J background and were backcrossed to C57BL/6Uni (imported from Zentralinstitut fuer Versuchstierzucht, Hannover, Germany) for three generations (N3), as further backcrossing caused infertility. Therefore, mixed background was maintained in the population by preferentially mating animals that were not closely related. Ten different breeding pairs of heterozygous mice were intercrossed to generate the wild-type (wt) and homozygous knockdown littermates $\left(\right.$ VAChT $\mathrm{KD}^{\mathrm{HOM}}$ ) used in this study. Male wild-type and VAChT KD ${ }^{\mathrm{HOM}}$ littermates were maintained in mixed groups of 3-5 animals in the animal facility at UWO according to the federal and provincial guidelines for the care and use of animals. They were housed in a temperature controlled room and a 12/12h light-dark cycle with light on/off at 7 am/pm and food and water ad libitum. Animals were tested during the light cycle, and age ranged from 2 to 5 months at testing. All experimental protocols were approved by the UWO Animal Use Subcommittee.

Behavioural Testing: For testing of startle responses four startle boxes (Med Associates) were used. For an input/output function of the startle amplitude depending on startle stimulus 
intensity, 12 VAChT KD ${ }^{\mathrm{HOM}}$ and 14 wild-type animals were used. PPI and habituation were tested using 16 wild-type and 16 VAChT KD ${ }^{\mathrm{HOM}}$ mice. Animals were pseudo-randomly assigned to one of the boxes. Two days prior to the start of experiments mice were acclimated to the startle boxes twice per day for 3-5 min with background noise but without stimulation. For measuring habituation and PPI, 3 startle stimuli were presented in the last acclimation session and startle responses recorded for determination of the individual optimum gain factors set for each animal. This was necessary since absolute startle response amplitudes differed substantially between individual animals. Low startle responses were close to the noise level at the default gain setting, whereas startle responses in other mice overshot the measuring range (see figure 2). The gain factors were then maintained for each animal throughout all subsequent behavioural sessions. The STARTLE software (Med Associates) was used to control experiments. During a behavioural session, animals first exposed to a $\mathbf{5}$ min. acclimation period with a background noise of $65 \mathrm{~dB}$. Two experimental blocks followed: in block I, 30 startle stimuli of $20 \mathrm{~ms}$ duration and $105 \mathrm{~dB}$ white noise were presented every $20 \mathrm{sec}$ in order to measure short-term habituation. In block II, 70 startle stimuli were presented preceded (or not) by a 4 ms prepulse of $80 \mathrm{~dB}$ white noise with 6 different interstimulus intervals (ISIs): 0, 6, 12, 20, 50, 100, and 250 ms. Each ISI trial was presented ten times in a pseudo-randomized order. The 0 ms ISI trials (no prepulse) were measured to assess baseline-startle responses. The startle stimulus amplitude of $105 \mathrm{~dB}$ was chosen since this is the intensity where startle responses reach the maximum amplitude (see figure 2). The different ISI were used to address the hypothesis that different receptors mediate PPI at different ISIs (see discussion). The behavioural testing was repeated on 5 subsequent days at around the same time of the day (see supplementary figure). 
For rescue experiments, 32 (pre-test injections) and 19 (post-test injections) VAChT KD ${ }^{\mathrm{HOM}}$ mice, as well as 14 wild-type mice were randomly assigned for control (saline) injections or galantamine (1 mg/kg i.p.). For pre-test injections animals were injected and returned to their home cage. About 10 min later they were placed into startle boxes. For post-test injections, animals were removed from startle boxes after the testing, returned to their home cage and were then immediately injected (see figure 1). Only the acclimation period and block I were executed in the rescue experiments.

Data Analysis: For the analysis of PPI, the ten 0 ms ISI trials per mouse in block II were averaged and set as baseline startle (100\%). The ten trials per all the other ISIs were also averaged and expressed as percentage of the respective baseline startle (startle amplitude * 100/baseline startle amplitude). The data was then averaged between animals of each genotype and error bars indicate SEM between animals of the same group.

For analysis of short-term habituation (STH), the 30 startle responses of block I were normalized to the average of the first two startle responses of each mouse, averaged over all five days and mice per genotype and plotted.

For analysis of long-term habituation (LTH), the averages of the first three startle responses in block 1 were calculated for each animal per day, averaged over mice per genotype/treatment, and plotted over the five days of testing. Only the first three startle responses were taken to exclude possible effects on STH in LTH analysis. For the diagrams showing the time course of habituation over 5 days, the whole data of block I was grouped in blocks of six subsequent startle responses in order to avoid noise.

Unpaired or paired student's t-tests or two way ANOVAs with repeated measurements and a mixed data design were used to assess statistical significant differences. Factor 1 was always the 
genotype or drug treatment (in rescue experiments), factor 2 was the ISI in the PPI experiments, the trial number in STH experiments, and the number of the day in LTH experiments. The Microsoft Excel add-in program StatEL (ad Science, France) was used for statistical analysis.

\section{Results:}

\section{Genotypes did not differ in startle amplitude}

14 wild-type and 12 VAChT $\mathrm{KD}^{\mathrm{HOM}}$ mice were tested for their startle response amplitude with increasing startle stimulus intensities from $65 \mathrm{~dB}$ background to $120 \mathrm{~dB}$ white noise in $5 \mathrm{~dB}$ steps. All startle boxes were calibrated to box 1 in order to allow for a direct comparison of startle amplitudes. As shown in figures 2A and 2B, the absolute startle response amplitude differed substantially between individual mice with high, intermediate, and low startler in both groups. A two-way repeated measurement ANOVA with genotype as independent factor and stimulus amplitude as dependent factor revealed no effect of the genotype on startle response amplitudes $(F(1,311)=0.08, p=0.78)$, a significant effect of the startle stimulus amplitude $(\mathrm{F} 11,301)=17.21, \mathrm{p}<0.00001$, and no interaction of the two factors $(\mathrm{p}=0.33)$. Whereas high startler overshot the measuring scale, low startler did barely raise the signal above the noise level, making it difficult to quantify startle attenuation by PPI or habituation. We therefore set fixed gain factors for the platform signal of each animal in all subsequent experiments: gain factors for the measured startle response in animals that overshoot at $105 \mathrm{~dB}$ in a gain setting pre-test (see figure 1 and Material \& Methods) were reduced from the default gain of 2 to a gain of 1.5. Intermediate startlers kept the gain of 2, low startlers were measured with a gain of 4 in order to increase the signal to noise level in these animals. 
The averages of the three startle amplitudes of each animal from the gain setting pre-tests of the following experiments were also used to further compare for absolute startle responses amplitudes between genotypes. A students t-test (two-tailed, unpaired) indicated no difference in baseline startle response amplitudes between genotypes ( $\mathrm{p}=0.31, \mathrm{n}=16$ per group).

\section{Prepulse inhibition of startle}

Prepulse inhibition of startle was tested for different ISIs. As shown in figure 3, VAChT KD ${ }^{\mathrm{HOM}}$ mice and their wild-type littermates did not differ in their ability to suppress startle stimuli preceded by a prepulse [ANOVA factor genotype: $F(1,58)=0.82, p=0.78$ ]. Both groups showed an inhibition of startle by around $70 \%$ at ISIs between 5 and $50 \mathrm{~ms}$, with a maximum PPI of 75\% at 50 ms ISI. PPI slightly decreased in both groups at ISIs of $100 \mathrm{~ms}$ (66\% for wt, 67\% for KD) and $250 \mathrm{~ms}$ [50\% wt and 54\% KD, ANOVA factor ISI: $\mathrm{F}(5,58)=11.46, \mathrm{p}<0.0001$, no interaction between factors: $F(5,58)=0.84, p=0.84]$.

\section{Habituation}

Animals were habituated to startle stimuli during the 30 startle trials in block I before they were tested for PPI. As shown in figure 4a, both groups of animals habituated to 70-75\% percent of their initial startle amplitude (as measured as the average of the first two responses) within this testing session. ANOVA revealed a significant effect of trial number $[\mathrm{F}(29,270)=2.98$, $\mathrm{p}<0.0001]$, with no interaction of genotype and trial number effect $[F(20,270)=0.75, p=0.82]$, confirming that the genotype did not affect short-term habituation. 
We then tested for long-term habituation by analyzing the average of the first three startle responses over five days. As shown in figure 4b, initial startle responses declined over the course of five days to around $50 \%$ of the amplitudes in day 1 in wild-type mice. In contrast, mutant mice did not show any long-term habituation between sessions. ANOVA revealed a significant interaction of the factor genotype and day $[\mathrm{F}(4,145)=2.518, \mathrm{p}=0.045]$. The effect is summarized in figure 4c, where the courses of short- and long-term habituation over the five consecutive days are plotted for each genotype. Both genotypes still show STH, but only the wild-type mice show LTH, whereas the mutant mice startle to the same extent at the beginning of each test session.

\section{Rescue of the habituation deficit}

We next tested if we could rescue LTH by galantamine injections into VAChT $\mathrm{KD}^{\mathrm{HOM}}$ mice (1mg/kg i.p.) prior to startle experiments. For this experiment, half of 14 wild-type and 37 VAChT KD ${ }^{\mathrm{HOM}}$ mice were injected with saline and half with galantamine before the gain setting test. A two way ANOVA with genotype and injection as independent factors was performed on the average of the three startle responses of the gain setting test (equal gains for all groups). It did not detect any difference between genotypes or type of injection (genotype: $F(1,13)=0.03, p=0.86$; injection: $F(1,13)=3.93, p=0.06$; interaction: $F(1,13)=2.74, p=0.11)$. In order to further exclude the possibility that galantamine injections cause a general potentiation of startle responses, 16 wild-type and $16 \mathrm{VAChT} \mathrm{KD}^{\mathrm{HOM}}$ mice were injected with saline after two days acclimation to the boxes and startle was tested. On the next day, they received a galantamine injection prior to testing startle responses (figure 5a). A paired t-test showed no difference in baseline startle amplitudes between saline and galantamine injections in wild-type 
$(\mathrm{p}=0.23)$ or VAChT $\mathrm{KD}^{\mathrm{HOM}}$ mice $(\mathrm{p}=0.27)$, which matched the result of a mixed two way ANOVA with genotype as independent and injection as dependent group. [Injection effect: $F(1,23)=0.22, p=0.64)$.

For the rescue experiment, we injected the animals over five days, prior to behavioural testing. In wild-type animals, a mixed repeated measure ANOVA with injection as independent and trial or testing day as within group factor revealed that there was no effect of injection group on short-term habituation [interaction injection with trial: $\mathrm{F}(29,390)=1.08, \mathrm{p}=0.35$, figure $5 \mathrm{~b}$ ], and no effect of repeated galantamine injection over 5 days on long-term habituation [interaction injection with testing day: $F(4,66)=1.54, p=0.2$, figure $5 c]$ when compared to saline injected animals. We also tested for a difference between injection groups for days 1-5 using a paired student's t-test, which didn't reveal any differences between saline and galantamine injections in wild-type mice either $(\mathbf{p}=\mathbf{0 . 2 8 - 0 . 4 5})$. Please note that both injection groups seemed to show less STH and LTH than in the previous experiment without injections, probably due to the aversive effects of the injection procedure itself (compare figure $5 \mathrm{~b}-\mathrm{c}$ with figure $4 \mathrm{a}$ c). In VAChT KD ${ }^{\mathrm{HOM}}$ mice, a mixed repeated measure ANOVA with injection as independent and trial or testing day as within group factor revealed there was also no effect of injection on short-term habituation [interaction of factor injection with trial: $\mathrm{F}(29,270)=0.70, \mathrm{p}=0.87$, figure 5b]. However, galantamine injected VAChT KD ${ }^{\mathrm{HOM}}$ mice showed a decline of their average startle responses between days, whereas saline injected animals did not show any LTH, confirming the lack of LTH in VAChT KD ${ }^{\mathrm{HOM}}$ mice in a second cohort of mice (figure $5 \mathrm{~d}$ ). ANOVA confirmed a trend for a difference for an interaction treatment with day $[F(4,22)=1.33, p=0.054]$. Unpaired student's t-tests confirmed no difference between 
injection groups at days $1(p=0.39), 2(p=0.08)$ and $4(p=0.08)$, but a significant difference between injection groups at days $3(p=0.01)$ and $5(p=0.02)$.

In order to test whether the esterase inhibitor has to be present during learning/retrieval or if it is sufficient if it is present during the consolidation phase following learning we repeated the rescue experiment with the VAChT $\mathrm{KD}^{\mathrm{HOM}}$ mice, but administered galantamine (or saline) immediately after the behavioural testing. As expected, short-term habituation in this group of animals was again not affected by the type of injections and animals showed STH comparable to non-injected animals (figure 6a, compare fig. 4a). As shown in fig. 6b and c, however, galantamine failed to rescue LTH when injected immediately after the behavioural testing session [ANOVA interaction factors treatment and day: $\mathrm{F}(1,159)=0.43, \mathrm{p}=0.79]$.

\section{Discussion}

VAChT KD ${ }^{\mathrm{HOM}}$ mice have a 70\% reduced expression of VAChT resulting in reduced cholinergic neurotransmission. These mice show a decreased ability to maintain physical activity when a prolonged motor function is required such as in the wire-hang test or in a rotarod test, whereas heterozygous VAChT KD ${ }^{\mathrm{HOM}}$ mice with a $40 \%$ reduction of VAChT immunoreactivity are slower in motor learning than their wild-type controls, show impaired object and social recognition, but their motor function, olfaction, and spatial memory is mainly unperturbed (De Castro et al., 2009, Prado et al., 2006). We here show that VAChT KD ${ }^{\mathrm{HOM}}$ mice have normal PPI and STH of startle, but disrupted LTH. Surprisingly, our data show that homozygous VAChT KD ${ }^{\text {HOM }}$ mice do not exhibit lower startle responses than their wild-type littermates as revealed by comparison of the i/o relation (figure 2) and the comparison of the pre-test data. 
Small differences between genotypes, however, would be very difficult to detect given the huge variability of startle responses between animals of the same genotype. It still seems to be safe to state that the previously reported motor deficits of the VAChT $\mathrm{KD}^{\mathrm{HOM}}$ did not have a major impact on our results. The short startle responses every 20 sec may not be sufficient to deplete the cholinergic synaptic vesicles. Indeed, the reported motor deficits are specific for prolonged motor function, whereas initial synaptic release and release probability have been shown to be normal in these mice (Prado et al., 2006).

\section{Prepulse inhibition}

The inhibitory effect of a prepulse on the startle reflex is assumed to occur through cholinergic PPT projections to the PnC, but descending limbic cortico-striato-pallido-pontine circuitry is known to regulate the pontine inhibitory tone. This regulation determines the degree to which the prepulse can inhibit the subsequent startle response. The cholinergic system closely interacts with dopaminergic systems in striatum therefore it is not clear to what extent cholinergic drugs affect the PPI circuitry directly as opposed to PPI modulation through alterations in dopaminergic signalling. The expression of normal PPI in VAChT KD ${ }^{\mathrm{HOM}}$ mice was a surprising result, given the large body of evidence for an important role of cholinergic projections in PPI and the reported effects of systemic cholinergic drugs. We tested PPI with different interstimulus intervals between prepulse and startle stimuli, since it has been hypothesized that different transmitter receptors mediate PPI at different time scales (Fendt et al., 2001b, Jones \& Shannon, 2000a, Jones \& Shannon, 2000b, Yeomans et al.). We chose the prepulse intensity that yields maximum PPI without causing a startle reaction itself ( $85 \mathrm{~dB})$, and we varied the ISI from the 
commonly used $100 \mathrm{~ms}$, to 30 and $50 \mathrm{~ms}$, which are the ISIs yielding maximum PPI in mice, plus a very short and a long ISI, in order to make sure that we don’t miss any cholinergic contribution. There was no PPI deficiency at any ISI. However, as with the lack of an effect on baseline startle, we cannot exclude the possibility that although cholinergic transmission is reduced in the mutant mice, it still might be sufficient for PPI signalling in response to a short prepulse every 20 seconds. Furthermore, other neurotransmitter systems contributing to PPI, such as GABA (Fendt, 1999, Kodsi \& Swerdlow, 1995, Yeomans et al.), could be up-regulated and be compensating for the lack of cholinergic transmission in these mice. The unperturbed PPI in the mutant mice can therefore not lead to the assumption that ACh plays no role in mediating and modulating PPI.

\section{Habituation}

Short-term habituation (STH) of startle is assumed to occur within the primary startle pathway which is glutamatergic. More specifically, the glutamatergic synaptic terminals of sensory afferent fibres projecting on the startle mediating giant neurons in the PnC are assumed to undergo synaptic depression during STH (Schmid et al., 2010, Simons-Weidenmaier et al., 2006, Weber et al., 2002). The role of cholinergic modulation in habituation had been investigated in the past and it was assumed that STH to external stimuli is not directly modulated by cholinergic neurotransmission (Hughes, 1984), which is in accordance with our results. The disruption of STH in both treatment groups in the rescue experiment with pre-test injections is likely due to the aversive procedure of i.p. injections immediately prior to behavioural testing. Aversive stimuli can cause sensitization and fear potentiation of startle that would override the habituation 
process. The fact that STH is normal in both groups in the post-test injection rescue experiment supports this conclusion.

Galantamine injections did not enhance baseline startle responses. Although it prolongs the effect of acetylcholine, it might not affect the initial startle response amplitude, but rather the duration. Most importantly, pre-test galantamine injections seemed to restore LTH in VAChT KD ${ }^{\mathrm{HOM}}$ mice, although ANOVA failed to show a clear significance with $\mathrm{p}$ values just slightly above 0.05 . A paired t-test confirmed a significant difference in startle amplitudes between galantamine and saline injected VAChT KD ${ }^{\mathrm{HOM}}$ mice for days 3 and 5. This rescue experiment is difficult to perform, since the injection procedure itself leads to sensitization, opposing habituation in the following startle test (compare STH and LTH in figures 4 and 5), and decreasing the differences between wild-type and VAChT KD ${ }^{\text {HOM }}$ mice in terms of habituation. LTH disruptions could be rescued only by pre-test injections of galantamine, when galantamine was present during acquisition and expression of LTH. Unfortunately, unlike in many other learning paradigms, acquisition and expression of learning are difficult to separate in LTH experiments. Galantamine did not restore LTH when present during consolidation only (post-test injections).

Little is known about mechanisms underlying long-term habituation (LTH) of startle. LTH can be disrupted by lesions of the cerebellar vermis (Leaton \& Supple, 1986, Leaton \& Supple, 1991), thus it is an extrinsic modulation of startle that employs cellular substrates different from the ones that mediate startle and STH. There is evidence for a cholinergic innervation of the cerebellum (Jaarsma et al., 1997), but it's connection to the vermis or to LTH is unclear. While the cerebellar vermis is important for LTH, the cholinergic PPT projections to the PnC might be a downstream effector mediating the inhibition of startle during LTH. An increase of the 
cholinergic tone in this projection could mediate LTH. Interestingly, the first injection of galantamine seemed to already induce maximum LTH, as opposed to the gradual startle decline during normal LTH. This would be expected, if galantamine strongly enhances cholinergic activity as opposed to a gradual increase in cholinergic tone during LTH. A tonic cholinergic function would also be more vulnerable to reduced cholinergic neurotransmission in VAChT $\mathrm{KD}^{\mathrm{HOM}}$ mice, as opposed to a transient cholinergic activation during PPI.

Interestingly, the lack of LTH to startle stimuli in VAChT $\mathrm{KD}^{\mathrm{HOM}}$ mice is paralleled by a lack of habituation to a juvenile intruder in these mice (Prado et al., 2006). Moreover, mutant mice show a deficit in object recognition, which could also be explain by a lack of habituation to the recently explored object. It will be crucial to gain more information about the respective underlying circuitry and mechanisms in order to elucidate in what way these disruptions are connected to each other.

In conclusion, while PPI and STH were not impaired, the unexpected disruption of LTH in VAChT KD ${ }^{\mathrm{HOM}}$ mice gives us valuable insights into both, cognitive functions of cholinergic neurotransmission as well as mechanisms underlying LTH of startle. Future experiments will address whether the LTH deficit is specifically mediated by the cholinergic dysfunction in the midbrain (i.e. PPT) or by higher brain areas modulated by the basal forebrain cholinergic cell groups, using brain region selective regional knock-outs for VAChT. 


\section{References:}

Acri, J.B., Morse, D.E., Popke, E.J. \& Grunberg, N.E. (1994) Nicotine increases sensory gating measured as inhibition of the acoustic startle reflex in rats. Psychopharmacology (Berl), 114, 369-374.

Barak, S. (2009) Modeling cholinergic aspects of schizophrenia: focus on the antimuscarinic syndrome. Behav Brain Res, 204, 335-351.

Blaha, C.D., Allen, L.F., Das, S., Inglis, W.L., Latimer, M.P., Vincent, S.R. \& Winn, P. (1996) Modulation of dopamine efflux in the nucleus accumbens after cholinergic stimulation of the ventral tegmental area in intact, pedunculopontine tegmental nucleus-lesioned, and laterodorsal tegmental nucleus-lesioned rats. J Neurosci, 16, 714-722.

Bosch, D. \& Schmid, S. (2006) Activation of muscarinic cholinergic receptors inhibits giant neurones in the caudal pontine reticular nucleus. Eur J Neurosci, 24, 1967-1975.

Bosch, D. \& Schmid, S. (2008) Cholinergic mechanism underlying prepulse inhibition of the startle response in rats. Neuroscience, 155, 326-335.

Curzon, P., Kim, D.J. \& Decker, M.W. (1994) Effect of nicotine, lobeline, and mecamylamine on sensory gating in the rat. Pharmacol Biochem Behav, 49, 877-882.

Davis, M., Parisi, T., Gendelman, D.S., Tischler, M. \& Kehne, J.H. (1982) Habituation and sensitization of startle reflexes elicited electrically from the brainstem. Science, 218, 688690.

de Castro, B.M., Pereira, G.S., Magalhaes, V., Rossato, J.I., De Jaeger, X., Martins-Silva, C., Leles, B., Lima, P., Gomez, M.V., Gainetdinov, R.R., Caron, M.G., Izquierdo, I., Cammarota, M., Prado, V.F. \& Prado, M.A. (2009) Reduced expression of the vesicular acetylcholine transporter causes learning deficits in mice. Genes Brain Behav, 8, 23-35. 
Deutsch, S.I., Urbano, M.R., Neumann, S.A., Burket, J.A. \& Katz, E. Cholinergic abnormalities in autism: is there a rationale for selective nicotinic agonist interventions? Clin Neuropharmacol, 33, 114-120.

Felder, C.C., Porter, A.C., Skillman, T.L., Zhang, L., Bymaster, F.P., Nathanson, N.M., Hamilton, S.E., Gomeza, J., Wess, J. \& McKinzie, D.L. (2001) Elucidating the role of muscarinic receptors in psychosis. Life Sci, 68, 2605-2613.

Fendt, M. (1999) Enhancement of prepulse inhibition after blockade of GABA activity within the superior colliculus. Brain Res, 833, 81-85.

Fendt, M., Li, L. \& Yeomans, J.S. (2001a) Brain stem circuits mediating prepulse inhibition of the startle reflex. Psychopharmacology (Berl). pp. 216-224.

Fendt, M., Li, L. \& Yeomans, J.S. (2001b) Brain stem circuits mediating prepulse inhibition of the startle reflex. Psychopharmacology (Berl), 156, 216-224.

Hughes, R.N. (1984) Cholinergic and monoaminergic substrates of startle habituation. Life Sci, 34, 2101-2105.

Jaarsma, D., Ruigrok, T.J., Caffe, R., Cozzari, C., Levey, A.I., Mugnaini, E. \& Voogd, J. (1997) Cholinergic innervation and receptors in the cerebellum. Prog Brain Res, 114, 67-96.

Jones, C.K. \& Shannon, H.E. (2000a) Effects of scopolamine in comparison with apomorphine and phencyclidine on prepulse inhibition in rats. Eur J Pharmacol, 391, 105-112.

Jones, C.K. \& Shannon, H.E. (2000b) Muscarinic cholinergic modulation of prepulse inhibition of the acoustic startle reflex. J Pharmacol Exp Ther, 294, 1017-1023.

Koch, M., Kungel, M. \& Herbert, H. (1993) Cholinergic neurons in the pedunculopontine tegmental nucleus are involved in the mediation of prepulse inhibition of the acoustic startle response in the rat. Exp Brain Res, 97, 71-82. 
Kodsi, M.H. \& Swerdlow, N.R. (1995) Ventral pallidal GABA-A receptors regulate prepulse inhibition of acoustic startle. Brain Res, 684, 26-35.

Kumari, V., Antonova, E. \& Geyer, M.A. (2008) Prepulse inhibition and "psychosis-proneness" in healthy individuals: An fMRI study. Eur Psychiatry.

Kumari, V., Soni, W. \& Sharma, T. (2001) Influence of cigarette smoking on prepulse inhibition of the acoustic startle response in schizophrenia. Hum Psychopharmacol, 16, 321-326.

Kumari, V., Soni, W. \& Sharma, T. (2002) Prepulse inhibition of the startle response in risperidone-treated patients: comparison with typical antipsychotics. Schizophr Res, 55, 139-146.

Lara, A., Damasceno, D.D., Pires, R., Gros, R., Gomes, E.R., Gavioli, M., Lima, R.F., Guimaraes, D., Lima, P., Bueno, C.R., Jr., Vasconcelos, A., Roman-Campos, D., Menezes, C.A., Sirvente, R.A., Salemi, V.M., Mady, C., Caron, M.G., Ferreira, A.J., Brum, P.C., Resende, R.R., Cruz, J.S., Gomez, M.V., Prado, V.F., de Almeida, A.P., Prado, M.A. \& Guatimosim, S. Dysautonomia due to reduced cholinergic neurotransmission causes cardiac remodeling and heart failure. Mol Cell Biol, 30, 17461756.

Leaton, R.N. \& Supple, W.F., Jr. (1986) Cerebellar vermis: essential for long-term habituation of the acoustic startle response. Science, 232, 513-515.

Leaton, R.N. \& Supple, W.F., Jr. (1991) Medial cerebellum and long-term habituation of acoustic startle in rats. Behav Neurosci, 105, 804-816.

Lima Rde, F., Prado, V.F., Prado, M.A. \& Kushmerick, C. Quantal release of acetylcholine in mice with reduced levels of the vesicular acetylcholine transporter. $J$ Neurochem, 113, 943-951. 
Lopiano, L., de'Sperati, C. \& Montarolo, P.G. (1990) Long-term habituation of the acoustic startle response: role of the cerebellar vermis. Neuroscience, 35, 79-84.

Mesulam, M. (2004) The cholinergic lesion of Alzheimer's disease: pivotal factor or side show? Learn Mem, 11, 43-49.

Postma, P., Gray, J.A., Sharma, T., Geyer, M., Mehrotra, R., Das, M., Zachariah, E., Hines, M., Williams, S.C. \& Kumari, V. (2006) A behavioural and functional neuroimaging investigation into the effects of nicotine on sensorimotor gating in healthy subjects and persons with schizophrenia. Psychopharmacology (Berl), 184, 589-599.

Prado, M.A., Reis, R.A., Prado, V.F., de Mello, M.C., Gomez, M.V. \& de Mello, F.G. (2002) Regulation of acetylcholine synthesis and storage. Neurochem Int, 41, 291-299.

Prado, V.F., Martins-Silva, C., de Castro, B.M., Lima, R.F., Barros, D.M., Amaral, E., Ramsey, A.J., Sotnikova, T.D., Ramirez, M.R., Kim, H.G., Rossato, J.I., Koenen, J., Quan, H., Cota, V.R., Moraes, M.F., Gomez, M.V., Guatimosim, C., Wetsel, W.C., Kushmerick, C., Pereira, G.S., Gainetdinov, R.R., Izquierdo, I., Caron, M.G. \& Prado, M.A. (2006) Mice deficient for the vesicular acetylcholine transporter are myasthenic and have deficits in object and social recognition. Neuron, 51, 601-612.

Schmid, S., Brown, T., Simons-Weidenmaier, N.S., Weber, M. \& Fendt, M. (2010) Group III metabotropic glutamate receptors inhibit startle mediating giant neurons in the caudal pontine reticular nucleus but do not mediate synaptic depression/short-term habituation of startle. $J$ Neurosci, in press.

Semenova, S., Bespalov, A. \& Markou, A. (2003) Decreased prepulse inhibition during nicotine withdrawal in DBA/2J mice is reversed by nicotine self-administration. Eur J Pharmacol, 472, 99-110. 
Simons-Weidenmaier, N.S., Weber, M., Plappert, C.F., Pilz, P.K. \& Schmid, S. (2006) Synaptic depression and short-term habituation are located in the sensory part of the mammalian startle pathway. BMC Neurosci, 7, 38.

Steriade, M., Datta, S., Paré, D., Oakson, G. \& Curró Dossi, R.C. (1990) Neuronal activities in brain-stem cholinergic nuclei related to tonic activation processes in thalamocortical systems. J Neurosci. pp. 2541-2559.

Suemaru, K., Yasuda, K., Umeda, K., Araki, H., Shibata, K., Choshi, T., Hibino, S. \& Gomita, Y. (2004) Nicotine blocks apomorphine-induced disruption of prepulse inhibition of the acoustic startle in rats: possible involvement of central nicotinic alpha7 receptors. $\mathrm{Br} J$ Pharmacol, 142, 843-850.

Weber, M., Schnitzler, H.U. \& Schmid, S. (2002) Synaptic plasticity in the acoustic startle pathway: the neuronal basis for short-term habituation? Eur J Neurosci, 16, 1325-1332.

Yeomans, J.S. (1995) Role of tegmental cholinergic neurons in dopaminergic activation, antimuscarinic psychosis and schizophrenia. Neuropsychopharmacology, 12, 3-16.

Yeomans, J.S., Bosch, D., Alves, N., Daros, A., Ure, R.J. \& Schmid, S. GABA receptors and prepulse inhibition of acoustic startle in mice and rats. Eur J Neurosci, 31, 2053-2061. 


\section{Acknowledgements:}

This study was supported by grants from Natural Science and Engineering Council (NSERC) and the Ontario Mental Health Foundation (OMHF) awarded to S. Schmid, and from Canadian Institute for Health Research (CIHR) to V. Prado. 
Figure legends:

2-3 pre-testing days:

acclimation to box

$(2-3 \times 5 \mathrm{~min})$

gain-test

(5 min, 3 stimuli)

testing days 1- 5:

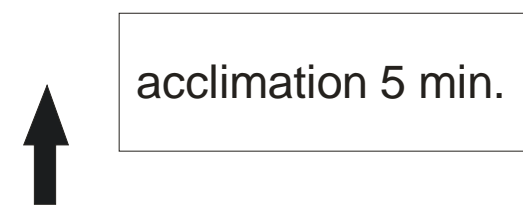

injection (pre-testing)
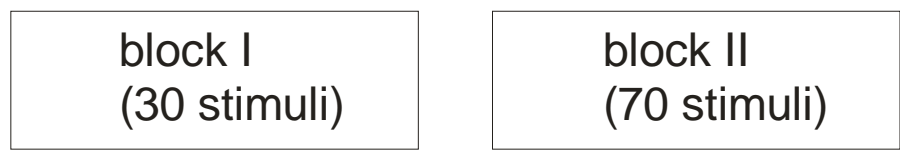

injection (post-testing)

Figure 1: Experimental scheme for testing habituation and PPI

All animals were acclimatized to the box 3-4 times for 5 min. During the last session they were exposed to three startle stimuli in order to set their individual gain factor. At testing day 1 , animals were placed into the startle box for $5 \mathrm{~min}$, then 30 startle stimuli were presented for habituation (block I). Subsequently, another 70 stimuli were presented with or without a prepulse in order to measure PPI. In the rescue experiments, injections were done on each of the five testing days either before animals were placed into the startle boxes (pre-testing), or right after testing when they were removed from the boxes (post-testing). For more details, please see the Materials and Methods section. 


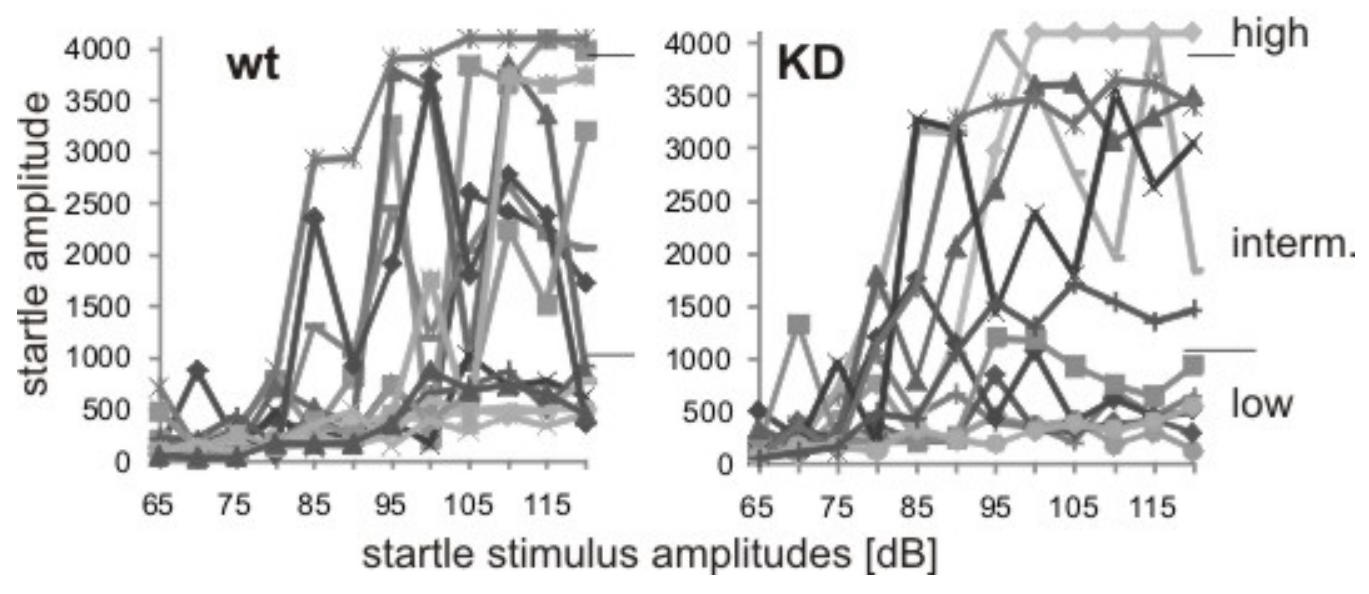

Figure 2: Individual input/output functions-startle response amplitudes in dependence of startle stimulus amplitudes- of 14 wt and 12 VAChT $\mathrm{KD}^{\mathrm{HOM}}$ mice

After 2-3 acclimation sessions, animals were exposed to $65 \mathrm{~dB}$ background noise and increasing startle stimulus intensities from $65 \mathrm{~dB}$ to $120 \mathrm{~dB}$. No difference in startle response amplitudes between genotypes was observed. Both wild-type and knock-out mice reach the maximum startle amplitude at around $105 \mathrm{~dB}$, however, there is a considerable variability of maximum startle responses between individuals within both groups. While some mice overshoot the measuring range, others barely raised the signal above the noise level. In subsequent experiments, gain factors for the measured motor response were adjusted according to the following scheme: mice that overshoot are measured with a gain of 1.5, intermediate mice are measured with the default gain of 2, low startlers are measured with a gain of 4 . 


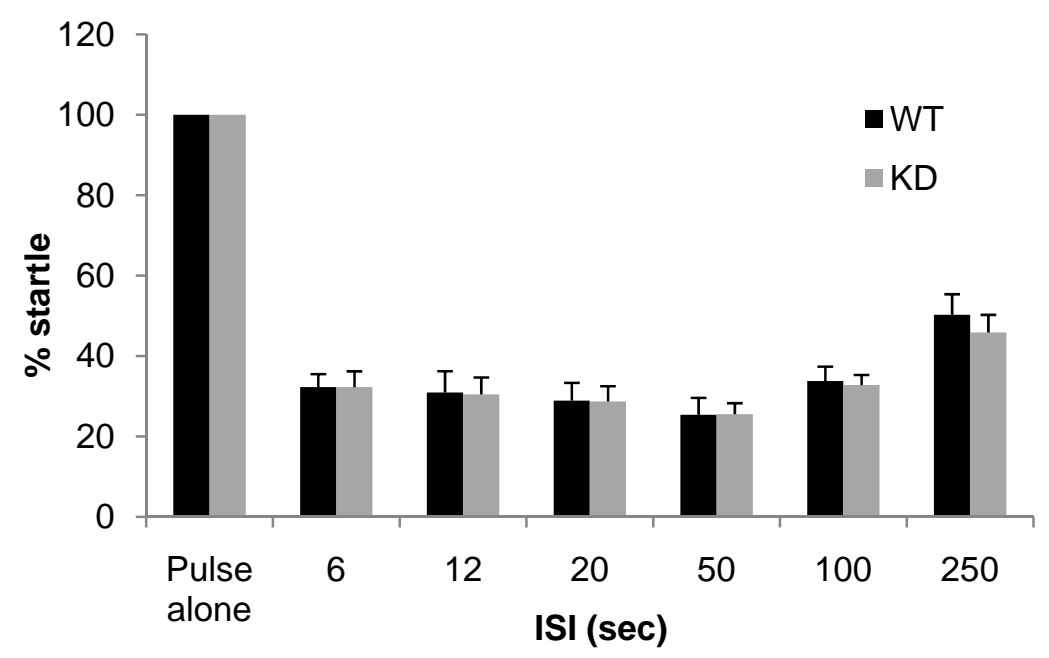

Fig. 3: Prepulse Inhibition

Startle response amplitudes to acoustic startle stimulus alone trials and startle stimuli preceded by an acoustic prepulse by different time intervals (ISI) as indicated. The average startle amplitude of each animal to startle stimulus alone trials was set to $100 \%$ and all other responses are expressed as percentage of it. Both genotypes, the VAChT $\mathrm{KD}^{\mathrm{HOM}}$ mice (KD) as well as their wild-type littermates (WT), showed an up to 75\% reduction of startle by the prepulse with no difference between groups ( $\mathrm{n}=16$ animals per genotype, error bars indicate standard error of mean (SEM)). 
a

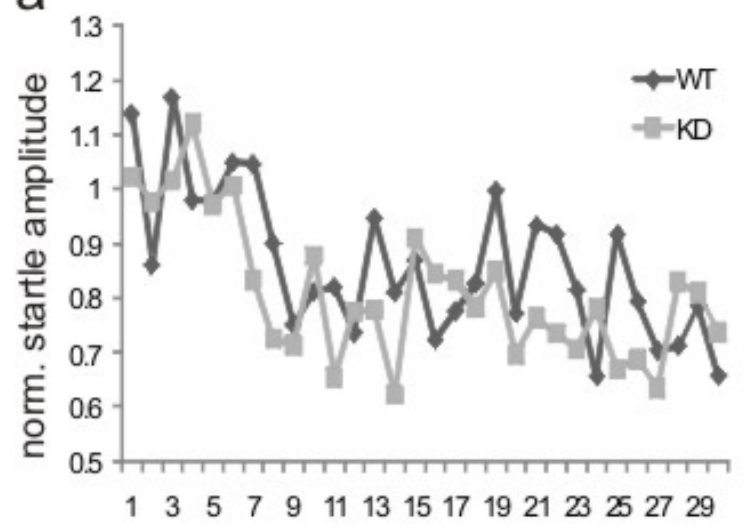

Trial No.

C

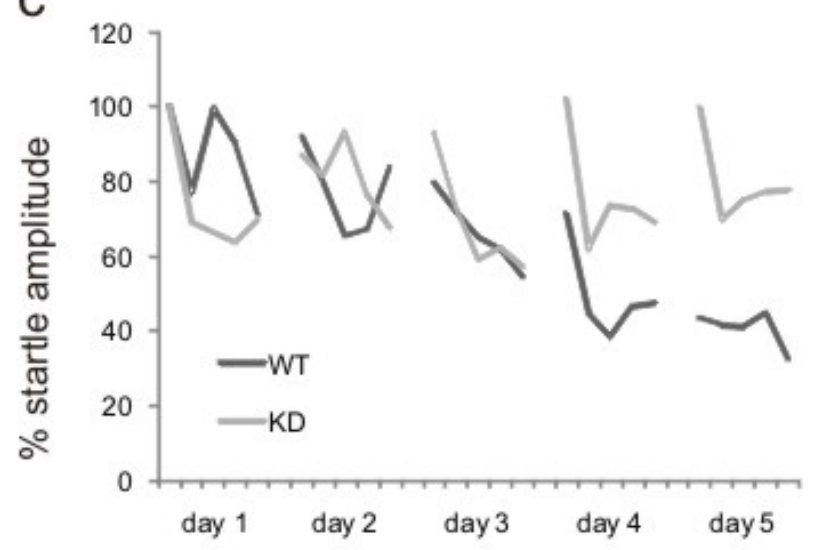

b

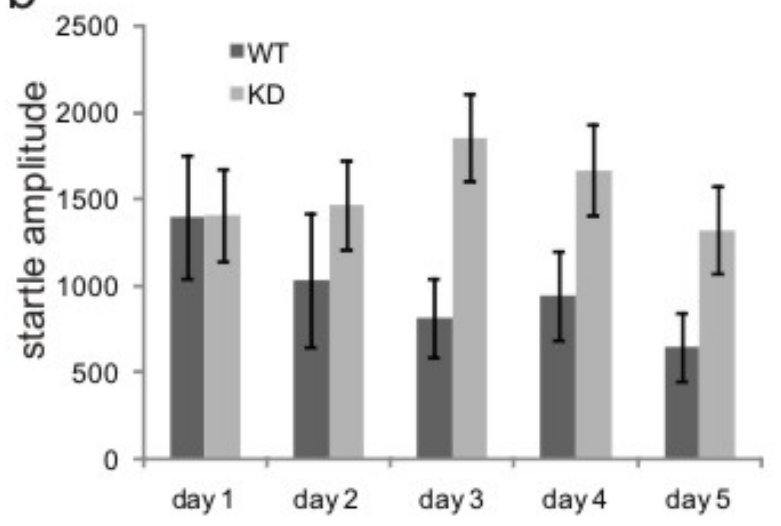

Figure 4: Habituation

a: Short-term (in session) habituation to 30 startle stimuli in VAChT $\mathrm{KD}^{\mathrm{HOM}}$ mice (KD) and their wild-type littermates (WT). Startle responses of each mouse of all five days were normalized to the average of its first two startle responses. Both groups show habituation to around $75 \%$ of their initial startle amplitudes.

b: Long-term (between sessions) habituation of VAChT $\mathrm{KD}^{\mathrm{HOM}}$ and wild-type mice. The averages of the first three startle responses of each day were calculated for each mouse and then averaged within genotypes. The startle responses declined over days in WT animals, but not in VAChT KD mice. Error bars indicate SEM. 
c: Course of short- and long-term habituation over five days. For more clarity, 6 consecutive startle responses of an animal were always averaged (blocks of 6). The data of each animal was then normalized to the value of the first block $(=100 \%)$. The graph shows that both genotypes show short-term habituation at every day, but whereas the overall startle amplitudes decline in wild-type animals over the 5 days, startle amplitudes remain at the same level in VAChT KD ${ }^{\mathrm{HOM}}$ mice, demonstrating the lack of long-term habituation ( $n=16$ animals per genotype in all experiments). 
a
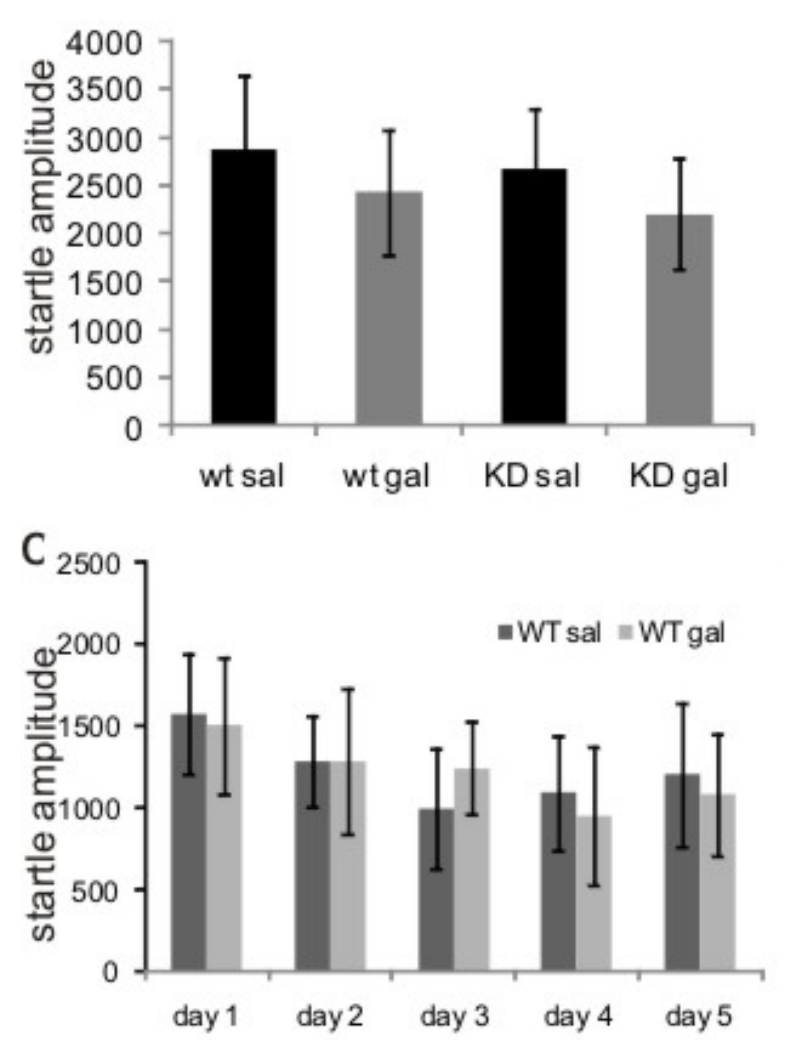

b

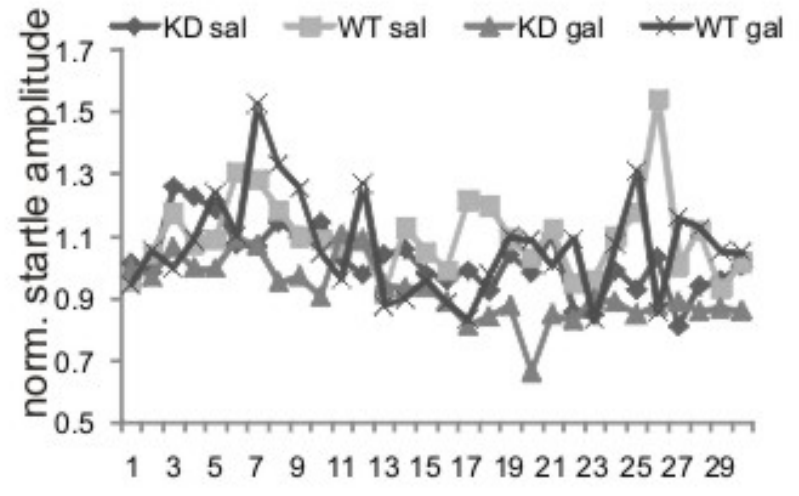

d

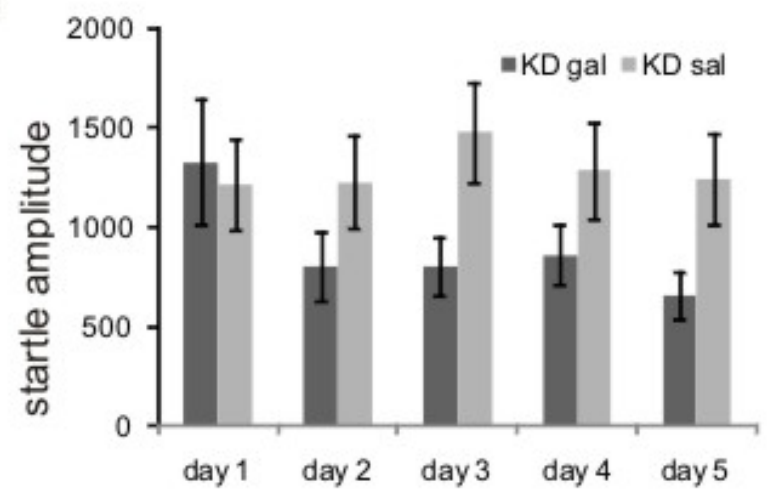

Figure 5: Partial rescue of long-term habituation by pre-test injections of galantamine

a: Control experiment testing the effect of galantamine versus saline on baseline startle responses. 16 wild-type and $16 \mathrm{VAChT} \mathrm{KD}^{\mathrm{HOM}}$ mice were acclimated to the boxes and underwent the pre-test. They then were exposed to acclimation and block I (habituation) in two subsequent days, with saline injection in day 1 and galantamine injection at day 2 . The first three startle responses of each animal after saline injection were compared to the first three responses 
after galantamine injections on the subsequent day. There was no effect of injections on baseline startle amplitudes in either genotype.

b: Short-term (in session) habituation to 30 startle stimuli in wild-type and VAChT $\mathrm{KD}^{\mathrm{HOM}}$ mice (WT and KD) injected by galantamine (gal) and in control by saline (sal). Startle responses of each mouse were normalized to the average of its first two startle responses. Short-term habituation was overlaid by initial sensitization in both genotypes (compare to figure 4a), probably due to the aversive process of injection (see Discussion). No differences between genotypes or injection groups could be detected ( $n=23$ wild-type and 27 KD mice).

c: Long-term (between sessions) habituation of wild-type mice following 5 days of saline or galantamine injections prior to behavioural testing. The averages of the first three startle responses of each day were calculated for each mouse and then averaged within treatment. No differences between injection groups could be detected at any of the five days ( $n=7$ wild-type mice each group).

d: Long-term habituation of VAChT KD ${ }^{\mathrm{HOM}}$ mice following 5 days of saline or galantamine injections prior to behavioural testing. VAChT $\mathrm{KD}^{\mathrm{HOM}}$ mice that were injected with galantamine showed a decline of their startle responses mainly after day 1 . Animals injected with saline did not show LTH, confirming the previous results (n= $18 \mathrm{KD}$ mice in the saline group and $19 \mathrm{KD}$ mice in the galantamine group). 
a

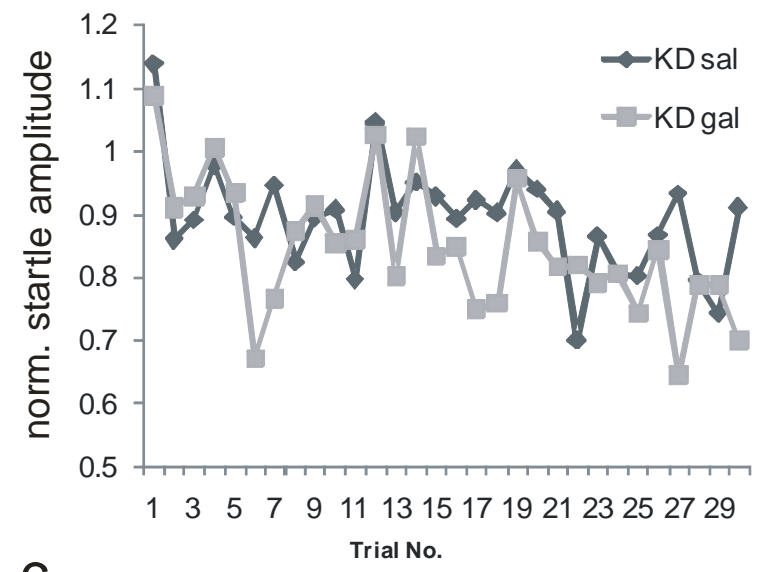

C

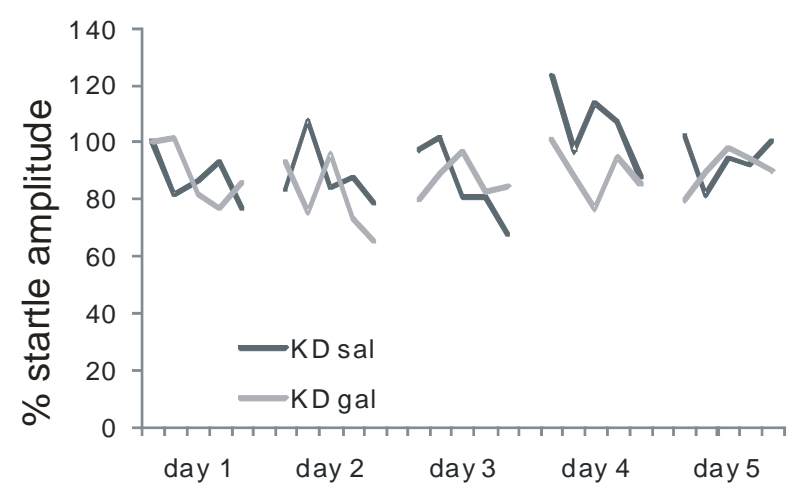

b

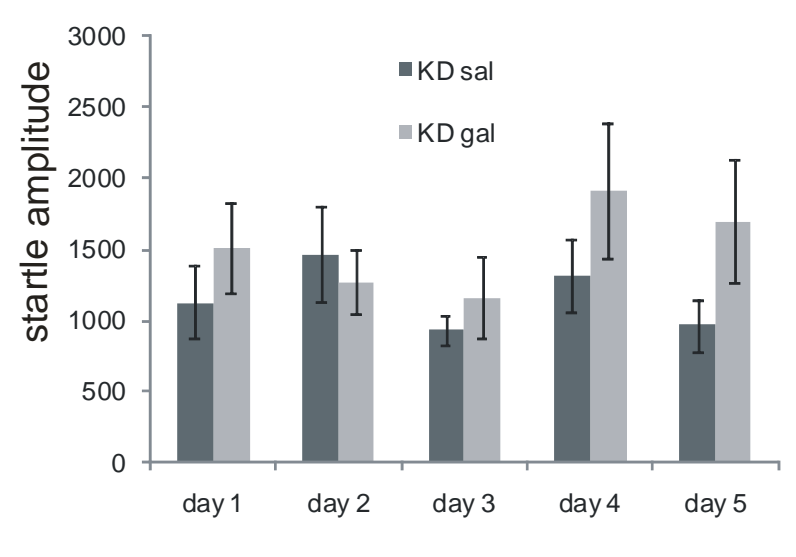

Figure 6: Post-test injections of galantamine

Animals were injected by $1 \mathrm{mg} / \mathrm{kg}$ i.p. galantamine immediately after behavioural testing.

a: Short-term (in session) habituation to 30 startle stimuli in VAChT $\mathrm{KD}^{\mathrm{HOM}}$ mice injected by galantamine (KD gal) and in control VAChT KD ${ }^{\mathrm{HOM}}$ mice injected by saline (KD sal). Startle responses of each mouse of all five days were normalized to the average of its first two startle responses. Both genotypes show intact short-term habituation (compare to figure 2A).

b: Long-term (between sessions) habituation of saline and galantamine injected mutant mice. The averages of the first three startle responses of each day were calculated for each mouse and 
averaged within treatment. Both groups of animals showed the same level of startle amplitudes over the five days.

c: Course of short- and long-term habituation over five days. For more clarity, 6 consecutive startle responses of an animal were always averaged (blocks of 6). The data of each animal was then normalized to the value of the first block $(=100 \%)$. Both groups of animals showed shortterm, but no long-term habituation over five days ( $\mathrm{n}=10 \mathrm{KD}$ mice with galantamine injections and $\mathrm{n}=9 \mathrm{KD}$ mice with saline injections). 